\title{
DAÑOS, EFECTOS Y AMENAZA DE TSUNAMIS EN AMÉRICA CENTRAL
}

\author{
Mario Fernández A. \\ Centro de Investigaciones Geofísicas (CIGEFI) y Red Sismológica Nacional (RSN: \\ ICE-UCR) Teléfono 2538407, Fax: 2532586 \\ mefernan@cariari.ucr.ac.
}

(Recibido 10/1/2000; Aceptado 30/5/2001)

\begin{abstract}
There have been 49 tsunamis in Central America since 1539 to 2000, 37 were in the Pacific and 12 in the Caribbean. Ten of them have a specific damage report, seven in the Pacific and the rest in the Caribbean. The total number of casualties due to local tsunamis is less than 500 but this number could be higher. The main earthquake sources related to the reported tsunamis are the Cocos-Caribbean subduction Zone, the Polochic-Motagua-Swan Fault System and the North Panama Deformed Belt.

An empirical estimation of tsunami hazard for Central America suggests that areas with higher tsunami potential in the Pacific are the coasts from Nicaragua to Guatemala and Central Costa Rica; on the Caribbean side, Honduras Gulf Zone and the coasts of Panama and Costa Rica have a major hazard. Earthquakes of magnitude larger than 7 with epicenters offshore or onshore (close to the coastline) could trigger tsunamis that would impact those zones.

RESUMEN: Desde 1539 hasta el presente han habido 49 tsunamis en América Central, 37 en el Pacífico y 12 en el Caribe; 10 de ellos tienen reportes de daños, siete en el Pacífico y tres en el Caribe. Estos tsunamis han cobrado cerca de 500 vidas humanas pero esta cifra podría ser mayor. Las fuentes más importantes de temblores tsunamigénicos de la región son: la zona de subducción Cocos-Caribe, el sistema de fallas Polochic-MotaguaSwan y el Cinturón Deformado del Norte de Panamá.

La estimación empírica de la amenaza por tsunamis sugiere que las áreas con mayor potencial tsunamigénico en la costa pacífica es el segmento que va desde Guatemala hasta Nicaragua y la parte central de Costa Rica y en el Caribe, el golfo de Honduras y la costas de Panamá y Costa Rica. Temblores de magnitud igual o mayor que 7,0 con epicentros en suelo marino o continental, cercano a la costa, podrían generar tsunamis que impacten las áreas en riesgo.
\end{abstract}

\section{INTRODUCCIÓN}

Más de 51000 residentes de localidades costeras murieron en el siglo XIX a causa de 94 tsunamis destructivos que ocurrieron a lo largo de las costas del océano Pacífico, en lo que se conoce como el Cinturón de Fuego del Pacífico. En tiempos más recientes, el gran temblor en el golfo Moro en Filipinas en agosto de 1976 produjo un tsunami que mató más de 8000 personas, el tsunami de la isla Flores en Indonesia que se llevó consigo más 
de 2000 vidas en 1992 y el que ocurrió en 1998 en Papúa Nueva Guinea, mató alrededor de 2000 personas. Estos datos muestran claramente que los tsunamis, como las erupciones volcánicas, los temblores, los deslizamientos y los huracanes, todavía están provocando grandes desastres y matando gente, lo cual es el más grave de sus efectos.

Los tsunamis se clasifican en tres categorías: 1) distantes, que se propagan a más de 750 $\mathrm{km}$ de su fuente; 2) regionales, que impactan pueblos costeros localizados a distancias variables entre 100 y $750 \mathrm{~km}$ a partir de la fuente y, 3) los locales, cuyos efectos no van más allá de los $100 \mathrm{~km}$ desde su lugar de origen. El alcance del tsunami suele ser proporcional al tamaño del temblor que lo originó, razón por la cual, cuanto mayor magnitud tenga el temblor, más grande podría ser la ola generada y mayor la distancia recorrida por la misma.

Las costas centroamericanas, como otras áreas en el mundo, están expuestas a los tres tipos de tsunamis, pero principalmente a los locales. El último tsunami del área, el de Nicaragua de 1992, es una real evidencia de la amenaza de tsunamis en esta activa zona sísmica. Aunque las costas centroamericanas han sido golpeadas por 34 tsunamis bien documentados, no había conciencia sobre la amenaza de tsunamis en la región antes del evento de Nicaragua. Fue justamente después de dicho tsunami que los científicos empezaron a considerar seriamente los efectos destructivos de los tsunamis de la región y algunas ideas surgieron para proteger a los pueblos costeros de Centroamérica. Fue así como en 1997 y bajo el auspicio del Centro para la Prevención de Desastres Naturales de América Central (CEPREDENAC), se inició una exhaustiva investigación sobre los tsunamis de la región, resultando un catálogo (Molina, 1997) que contiene datos de tsunamis históricos desde 1539 hasta el 2000 (actualizado), que estaban dispersos en diferentes fuentes. El presente artículo se basa en Fernández et al. (1999) y Fernández et al. (2000). Su resultado primordial es una estimación preliminar de la amenaza por tsunamis para la región, lo cual constituye el primer pa- so para el establecimiento de un confiable sistema de alerta contra tsunamis en el área.

\section{MARCO TECTÓNICO}

La región centroamericana y parte de las islas del Caribe, son parte de la placa Caribe. Esta placa se encuentra rodeada por las placas Cocos, Norteamericana, Nazca y Sudamericana (Fig. 1). Los tres límites tectónicos más importantes del área centroamericana son: La fosa Me-

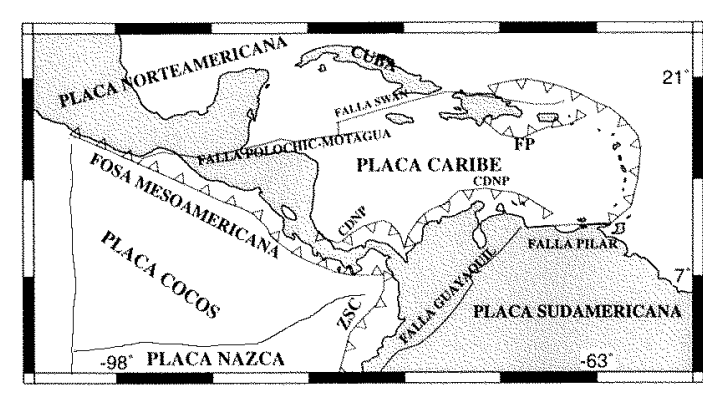

Fig. 1: Marco tectónico. FP: Fosa de Puerto Rico, CDNP: Cinturón Deformado del Norte de Panamá, ZSC: Zona de Subducción de Colombia (adaptado de Calais et al., 1992).

soamericana, sistema de fallas Polochic-Motagua-Swan y la Zona de Fractura de Panamá. Otro límite importante es el Cinturón Deformado del Norte de Panamá.

La fosa Mesoamericana es el límite entre la placa Caribe y la placa Cocos a lo largo del cual la primera se subduce bajo la segunda. Desde México hasta la parte central de Costa Rica el proceso de subducción es normal pero en el sur de Costa Rica la subducción llega a ser más superficial debido a la presencia de la cordillera submarina de Cocos, la cual colisionó con la placa Caribe hace aproximadamente 5 millones de años. El límite entre la placa Caribe y la placa Norteamericana es el sistema de falla Polochic-Motagua-Swan de movimiento trascurrente siniestral, este es un sistema que inicia en las áreas continentales de Guatemala y Honduras, cruza por el sur de Cuba y termina hacia el este de República Dominicana. La 
frontera entre la placa del Coco y la placa Nazca es lo que se conoce como Zona de Fractura de Panamá; esta zona está compuesta por fallas de orientación norte-sur ubicadas frente a la costa pacífica de Costa Rica y Panamá. El Cinturón deformado del Norte de Panamá (Bowin, 1976) es una irregularidad topográfica ubicada en el piso marino del Caribe, frente a la costa caribe de Panamá y Costa Rica; se ha propuesto que este rasgo topográfico es una zona de subducción dentro la placa Caribe.

\section{TSUNAMIS EN LA COSTA CARIBE}

En esta investigación se encontró que 12 tsunamis han golpeado pueblos costeros del Caribe desde 1539 hasta el 2000. Los datos principales de estos tsunamis así como los del temblor que los originó, se describen en el cuadro 1.

Los temblores que han causado tsunamis en la costa caribe de América Central son tanto del sistema de fallas Polochic-Motagua, como del Cinturón Deformado del Norte de Panamá. En la figura 2 se indican los temblores localizados que han originado tsunamis en el Caribe.
Son temblores de profundidad superficial, muchos de ellos con el epicentro en el área continental como el número 11 y el 12, que corresponden con los terremotos de Guatemala (1976) y Limón (1991), respectivamente. Estos datos dejan constancia de que no solamente los temblores originados en el piso oceánico disparan tsunamis. No obstante, por ocurrir el temblor en área continental, los tsunamis generados han sido muy pequeños y por ello, han causado poco daño. Cuatro de estos temblores ocurrieron en la zona de fallamiento inverso ubicado en la zona atlántica de Costa Rica. El cuadro 2 es un resumen de los daños y efectos más importantes de los 12 tsunamis en la costa caribe de América Central.

Tres de estos tsunamis tuvieron olas de altura mayor a $3 \mathrm{~m}$ y uno, mayores de $5 \mathrm{~m}$. Localidades enteras fueron destruidas por estos tsunamis. El único tsunami del Caribe con reporte de muertes es el que azotó el archipiélago de San Blas, frente a las costas de Panamá, en 1882. Es sorprendente que a pesar de que el tsunami de 1856 destruyó por completo la localidad de Omoa y otras localidades cercanas, no hubo reporte de muertos.

Cuadro 1

Temblores y tsunamis en la costa caribe de América Central

\begin{tabular}{|c|c|c|c|c|c|c|c|c|}
\hline \multirow[b]{2}{*}{ \# } & \multicolumn{5}{|c|}{ Datos del Temblor } & \multicolumn{3}{|c|}{ Datos del tsunami } \\
\hline & Fecha & Lat. $\mathrm{N}$ & Long. W & Mag. & Fuente & Localización & Alt.(m) & Mag. \\
\hline 1 & $1539-1124$ & & & & & Golfo Hond. & & \\
\hline 2 & $1798-0222$ & 10,2 & 82,9 & & $\mathrm{CV}$ & Matina, CR & & -1 \\
\hline 3 & $1822-0507$ & 09,5 & 83,0 & 7,6 & $\mathrm{CV}$ & Matina, CR & & -1 \\
\hline 4 & $1825-02 .$. & & & $5-5,5$ & & Golfo Hond. & & \\
\hline 5 & $1855-0925$ & & & $6-6,5$ & & Golfo Hond. & & \\
\hline 6 & $1856-0804$ & & & $7-8,0$ & & Golfo Hond. & 5 & 2 \\
\hline 7 & $1873-1014$ & 10,2 & 80,0 & & $\mathrm{CV}$ & Colón, Pan. & & \\
\hline 8 & $1882-0907$ & 10,0 & 79,0 & 7,9 & $\mathrm{CV}$ & San Blas, Pan. & 3 & 1 \\
\hline 9 & $1904-1220$ & 09,2 & 82,8 & 7,3 & AA & Bocas Toro & & \\
\hline 10 & $1916-0426$ & 09,2 & 83,1 & 6,9 & AA & Bocas Toro & 1,3 & 0 \\
\hline 11 & 1976-0204 & 15,2 & 89,2 & 7,5 & AA & Golfo Honduras & 0,45 & $-0,5$ \\
\hline 12 & $1991-0422$ & 09,6 & 83,2 & 7,6 & AA & Bocas Toro & 3 & 1 \\
\hline
\end{tabular}

CV: Camacho \& Víquez (1993), AA: Ambraseys \& Adams (1996) 
Cuadro 2

Daños, efectos y comentarios sobre los tsunamis del Caribe

\begin{tabular}{|c|c|c|}
\hline \# & Fecha & Daño, efectos, comentarios \\
\hline 1 & $1539-1124$ & Violenta sacudida sentida a bordo de un barco (Montessus de Ballore, 1888; Sutch, (1981). \\
\hline 2 & $1798-0222$ & El mar se agitó intensamente y produjo inundaciones (González, 1910; Peraldo \& Montero, 1994). \\
\hline 3 & $1822-0507$ & $\begin{array}{l}\text { Inundaciones por crecida en ríos y bahías. En Bocas del Toro, un residente mencionó la existencia } \\
\text { un tsunami (Montessus de Ballore, 1888; González, 1910; Montero, 1986; Camacho \& Víquez, 1993; } \\
\text { Boschini \& Montero, 1994). }\end{array}$ \\
\hline 4 & $1985-02$ & $\begin{array}{l}\text { Sacudida reportada por pasajeros a bordo de un barco. Fuerte sonido después del temblor (Montessus } \\
\text { de Ballore, 1888; Rockstroch, 1902; Sieberg, 1932; Sutch, 1981). }\end{array}$ \\
\hline 5 & $1855-0925$ & $\begin{array}{l}\text { El barco Simpronius fue repentinamente levantado, cayendo luego bruscamente (Montessus de } \\
\text { Ballore, 1888; Sutch, 1981). }\end{array}$ \\
\hline 6 & $1856-0804$ & $\begin{array}{l}\text { En Omoa, (Honduras), el mar bajó y luego subió hasta alcanzar una altura de } 5 \text { m, por lo que quedó } \\
\text { en ruinas. Hubo daños en Cortez, Atlántida y Trujillo. El tsunami consistió de varias olas que llegaron } \\
\text { una tras otra (Montessus de Ballore, 1888; Milne, 1912; Sieberg,1932; Heck, 1947; Montandon, } \\
\text { 1962; Iida et al., 1967; Sutch, 1981; Cruz \& Wyss, 1983; Soloviev \& Go, 1984). }\end{array}$ \\
\hline 7 & $1873-1014$ & $\begin{array}{l}\text { La oscilación causada por la ola fue sentida por personas a bordo de un barco que se encontraba en } \\
\text { puerto (Camacho \& Víquez, 1993). }\end{array}$ \\
\hline 8 & $1882-0907$ & $\begin{array}{l}\text { El temblor causó un tsunami que afectó el área de San Blas (Panamá), con olas de más de } 3 \text { m. } \\
\text { Murieron alrededor de } 100 \text { nativos. La ola avanzó gran distancia tierra adentro y a su regreso borró } \\
\text { las villas de las playas de las diferentes islas. En el Buque Honduras se sintió la oscilación del agua; } \\
\text { el mar empezó a moverse rápidamente y el barco se meció al ritmo de la ola. Antes de la sacudida se } \\
\text { escuchó un estruendo (Societé de Publications Periódiques de París, 1882; Montessus de Ballore, } \\
\text { 1888, Nelson, 1889; Milne, 1912; Sieberg, 1932; Tabor, 1967; lida, et al.,1967; Soloviev \& Go, 1984; } \\
\text { Mendoza \& Nishenko, 1989; Grases, 1990). }\end{array}$ \\
\hline 9 & $1904-1220$ & $\begin{array}{l}\text { Parte de la isleta Sapodilla subsidió. La tripulación del barco de guerra estadounidense Dixie, sintió } \\
\text { severamente el movimiento de las aguas (González, 1910; Camacho \& Víquez, 1993; Ambraseys \& } \\
\text { Adams, 1996). }\end{array}$ \\
\hline 10 & $1916-0426$ & $\begin{array}{l}\text { La ola, de más } 1 \text { m de alto, acarreó canoas y escombros } 200 \text { m tierra adentro y destruyó tanques de } \\
\text { almacenamiento. Inundó la localidad de Bastimentos, isla de Carenero (Kirkpatrick, 1920; Feldman, } \\
\text { 1984; Güendel, 1986; Víquez \& Toral, 1987; Camacho \& Víquez, 1992; Camacho \& Víquez, 1993; } \\
\text { Ambraseys \& Adams, 1996). }\end{array}$ \\
\hline 11 & 1976-0204 & $\begin{array}{l}\text { Un tsunami con amplitud máxima de } 45 \mathrm{~cm} \text { fue registrado en el mareógrafo de Puerto Cortés, } \\
\text { Honduras (Buckman et al., 1978; Young et al., 1989; Ambraseys \& Adams, 1996). }\end{array}$ \\
\hline 12 & $1991-0422$ & $\begin{array}{l}\text { Una ola de } 2 \text { m de alto fue registrada en San Cristóbal, Panamá. Afectó toda la costa desde el norte } \\
\text { de Limón hasta Panamá. Desde Cahuita hasta la isla Bastimentos se observó una ola de } 2 \text { y } 3 \text { m. } \\
\text { Inundó hasta } 200 \text { m tierra adentro, también fue registrada en Islas Vírgenes y Puerto Rico (Plafker } \\
\text { \& Ward, 1992; Denyer et al., 1992; Barquero \& Rojas, 1994; Camacho, 1994; Ambraseys \& } \\
\text { Adams, 1996). }\end{array}$ \\
\hline
\end{tabular}


TSUNAMIS DE LA COSTA PACÍFICA

Los tsunamis más grandes de América Central han ocurrido en la costa pacífica. Esto es normal considerando que la Fosa Mesoamericana, ubicada frente a dicha costa, es el lími- te tectónico con mayor actividad sísmica en la región. Según este estudio, 37 tsunamis han golpeado esta costa desde 1539 hasta el presente. En el cuadro 3 se resumen los parámetros más importantes de los tsunamis y de los temblores que los originaron.

Cuadro 3

Parámetros de los tsunamis y temblores asociados de la costa pacífica

\begin{tabular}{|c|c|c|c|c|c|c|c|c|}
\hline \multirow[b]{2}{*}{$\#$} & \multicolumn{5}{|c|}{ Datos del Temblor } & \multicolumn{3}{|c|}{ Datos del tsunami } \\
\hline & Fecha & Lat. $\mathrm{N}$ & Long. W & Mag. & Fuente & Localización & Alt.(m) & Mag. \\
\hline 1 & $1579-0316$ & & & & & Isla Caño, C.R. & & \\
\hline 2 & $1621-0502$ & 08,97 & 79,55 & & $\mathrm{VC}$ & La Vieja, La Vieja & & \\
\hline 3 & $1844-05 .$. & 11,20 & 84,80 & 7,4 & $\mathrm{R}$ & Lago Nicaragua (Seiche) & & \\
\hline 4 & $1854-0805$ & 08,50 & 83,00 & 7,3 & $\mathrm{R}$ & Golfo Dulce, C.R. & & 1,5 \\
\hline 5 & $1859-0826$ & 13,00 & 87,50 & 6,2 & $\mathrm{R}$ & Golfo Fonseca, Hond. & & 1,5 \\
\hline 6 & $1859-1209$ & 13,75 & 89,75 & 7,0 & SG & Acajutla, Salv. & & 1,5 \\
\hline 7 & $1884-105$ & 4,00 & 76,00 & 7,5 & $\mathrm{R}$ & Acandi, Colombia & & \\
\hline 8 & $1902-0118$ & 14,70 & 91,60 & 6,3 & $\mathrm{~A}$ & Ocos, Guatemala & & \\
\hline 9 & $1902-0226$ & 13,00 & 89,50 & 7,0 & $\mathrm{R}$ & Guatemal-Salv. & & 2 \\
\hline 10 & $1902-0419$ & 14,90 & 91,50 & 7,5 & AA & Ocos, Guatemala & & -1 \\
\hline 11 & $1904-0120$ & 07,00 & 82,00 & 7,0 & AA & & & \\
\hline 12 & $1905-0120$ & 09,85 & 84,68 & 6,8 & $\mathrm{R}$ & Isla del Coco, C.R. & & \\
\hline 13 & $1906-0131$ & 01,00 & 81,30 & 8,1 & PS & Ecuador, Panamá,, C..R. & $2-5$ & \\
\hline 14 & $1906-\ldots$. & & & & & El Salv. & & \\
\hline 15 & $1913-1002$ & 07,10 & 80,60 & 6,7 & AA & Azuero, Panamá & & -1 \\
\hline 16 & $1915-0907$ & 13,90 & 89,60 & 7,7 & AA & Costa Sur, Salv. & & 0,5 \\
\hline 17 & $1916-0131$ & & & & & Canal de Panamá & & \\
\hline 18 & $1916-0525$ & 12,00 & 90,00 & 7,5 & $\mathrm{H}$ & El Salvador & & \\
\hline 19 & $1919-0629$ & 13,50 & 87,50 & 6,7 & AA & Corinto, Nicaragua & & \\
\hline 20 & $1919-1212$ & & & & & El Ostial, Nicaragua & & \\
\hline 21 & $1920-1206$ & & & & & Golfo Fonseca, Hond. & & \\
\hline 22 & 1926-1105 & 12,30 & 85,80 & 7,0 & AA & Nicaragua & & \\
\hline 23 & $1934-0718$ & 08,10 & 82,60 & 7,5 & AA & Golfo Chiriquí, Pan. & 0,60 & 1,5 \\
\hline 24 & $1941-1205$ & 08,70 & 83,20 & 7,6 & $\mathrm{AA}$ & Dominical, C.R. & 0,22 & -1 \\
\hline 25 & $1941-1206$ & 10,00 & 85,20 & 6,9 & M & Golfo Nicoya, C.R. & 0,08 & -2 \\
\hline 26 & $1950-1005$ & 10,00 & 85,70 & 7,7 & AA & C.R., Nicaragua, Salv. & & -1 \\
\hline 27 & $1950-1023$ & 14,30 & 91,80 & 7,3 & AA & Guatemala-Salv. & & -1 \\
\hline 28 & $1951-0803$ & 13,00 & 87,50 & 6,0 & $\mathrm{~L}$ & Golfo Fonseca, Hond. & & \\
\hline 29 & $1952-0513$ & 10,30 & 85,30 & 7,0 & $\mathrm{CW}$ & Puntarenas, Costa Rica & 0,10 & -3 \\
\hline 30 & $1956-1024$ & 11,50 & 86,50 & 7,2 & AA & San Juan Sur, Nic. & & \\
\hline 31 & $1957-0310$ & 51,63 & 171,4 & 8,1 & PS & Acajutla, Salv. & $>2$ & \\
\hline 32 & $1960-0522$ & 38,20 & 73,50 & 8,5 & PS & Guatemala-Salv. & & \\
\hline 33 & $1962-0312$ & 08,10 & 82,90 & 6,7 & $\mathrm{CW}$ & Golfo Chiriquí, Pan. & 0,30 & -1 \\
\hline 34 & $1968-0925$ & 15,60 & 92,60 & 6,0 & BSSA & Guatemala & 1,9 & \\
\hline 35 & $1976-0711$ & 07,40 & 78,12 & 7,0 & $\mathrm{G}$ & Darién, Panamá & & -1 \\
\hline 36 & $1990-0325$ & 09,80 & 84,80 & 7,0 & AA & Puntarenas, C.R. & 1 & 0 \\
\hline 37 & 1992-0902 & 11,70 & 87,40 & 7,2 & $\mathrm{AA}$ & Nicaragua & 9,5 & 2,5 \\
\hline
\end{tabular}

Mag.: magnitud, VC: Víquez \& Camacho (1994), R: Rojas et al., (1993), SG: Soloviev \& Go (1984), A: Ambraseys (1995), AA: Ambraseys \& Adams (1996), PS: Pacheco \& Sykes (1992), H: Hatori (1995), L: Leeds (1974), CW: Cruz \& Wyss (1983), BSSA: Bull. Seismol. Soc. America, 1969; G: Grases (1990). 
Veintitrés tsunamis fueron disparados por temblores de subducción debido a la interacción entre las placas Cocos y Caribe, uno provino de la Zona de Fractura de Panamá, dos de fallas superficiales, uno de la interacción entre las placas Norteamericana y Sudamericana y seis más permanecen sin identificación de su ambiente tectónico. Con base en estos datos se concluye que el límite tectónico Cocos-Caribe es el ambiente tectónico más importante en la generación de temblores tsunamigénicos en América Central.

La ubicación epicentral de los temblores tsunamigénicos del Pacífico de América Central se muestra en la figura 2. Claramente se observa que los temblores tsunamigénicos del Pacífico se distribuyen a lo largo de toda la costa y no están concentrados, como los del Caribe. A lo largo de la costa pacífica, muchos de los temblores tsunamigénicos tienen su epicentro en el área continental o cerca de la costa, lo cual pudo haber contribuido a la reducción de la altura de la ola. Los tsunamis provocados por temblores con epicentro en el mar han sido los más destructivos, alcanzando el mayor 9,5 $\mathrm{m}$ de alto.

Tsunamis regionales de cualquier lugar del Cinturón de Fuego del Pacífico también han azotado la costa pacífica centroamericana. En 1957 un temblor de la Islas Aleutianas generó un tsunami que alcanzó las costas de El Salvador y causó extensivo daño a los pueblos costeros, llegando incluso a matar pobladores. El temblor de magnitud 8,5 que ocurrió en Chile en 1960, golpeó las costas de Guatemala y El Salvador, pero no se reportaron daños ni pérdidas de vidas a causa de este evento. También, un temblor de Tumaco (Colombia), provocó un tsunami que fue observado a lo largo de toda la costa pacífica de América Central pero no se reportaron daños. Un tsunami interesante es el que afectó el golfo de Fonseca en Honduras en 1951; los reportes históricos apuntan a un lahar que descendió del volcán Cosigüina de Nicaragua, como el evento disparador del tsunami (cuadro 4).

Estos tsunamis inundaron villas, arrastraron casas y produjeron gran daño a la propiedad privada, solamente 355 víctimas fueron reportadas. Sin embargo, este número podría ser mayor porque tres tsunamis (golfo Dulce Costa Rica, 1854; Pedasi, Panamá, 1913 y Acajutia, El Salvador, 1957) causaron gran daño, pero ningún número de muertes fue reportado. Además, el tsunami de la provincia de Darién, Panamá, de

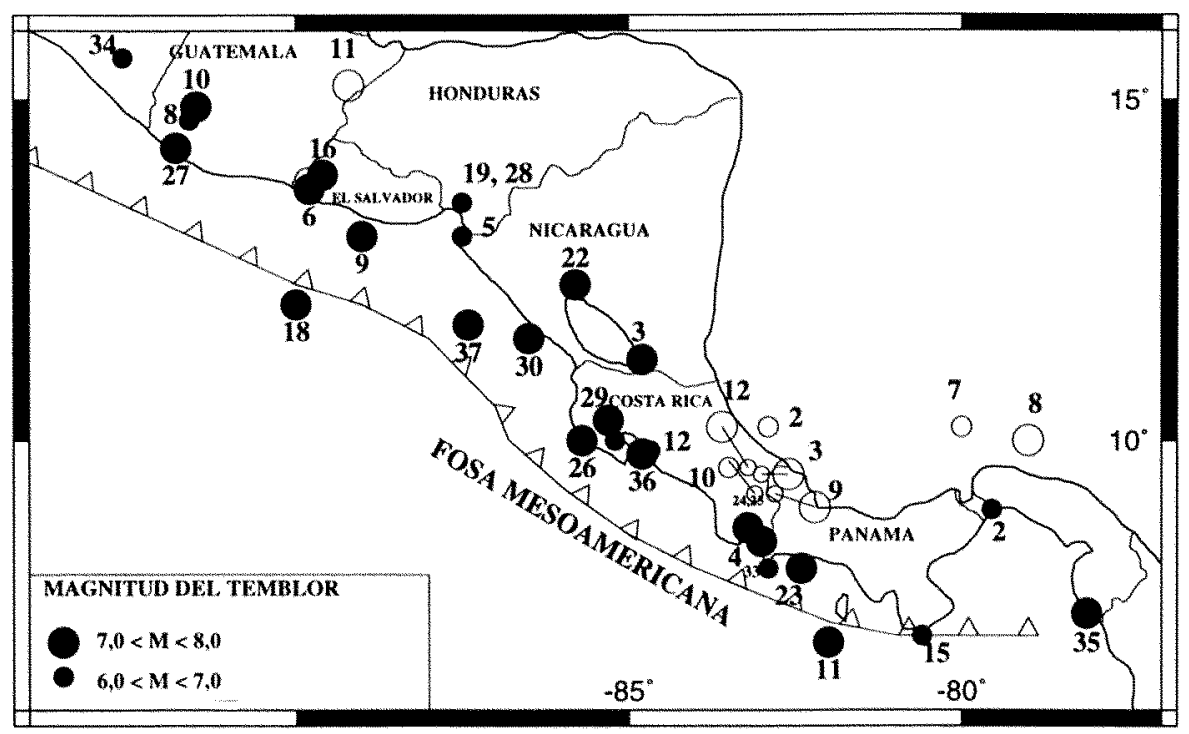

Fig. 2: Epicentros de los temblores tsunamigénicos. Los círculos de color negro son temblores que causaron tsunamis en el Pacífico y aquellos sin relleno (desplazados de su epicentro el cual es representado con los círculos pequeños) son sismos cuyos tsunamis pertenecen al Caribe. El número cercano al epicentro es el número del temblor en los cuadros 1 y 3. 
Cuadro 4

Daños, efectos y comentarios de los tsunamis en la costa pacífica

\begin{tabular}{lll}
\hline$\#$ & Fecha & Daños, efectos, comentarios \\
\hline 1 & $1579-0316$ & $\begin{array}{l}\text { Un barco fue mecido y vibró como si estuviera reposando en arena seca (González, 1910; Peraldo } \\
\text { \& Montero, 1994). }\end{array}$ \\
2 & Las olas casi inundan La Calle de las Carreras, Panamá (Víquez \& Camacho, 1994). \\
3 & 1621-0502 & $\begin{array}{l}\text { Dos ríos fueron reabiertos en Nicaragua, el lago de Nicaragua se desbordó (Montessus de Ballore, } \\
\text { 1888; Crawford, 1902; Milne, 1912; Montandon, 1962; Jorgensen, 1966; Leeds, 1974; Carr \& } \\
\end{array}$ \\
&
\end{tabular}
Soloviev \& Go, 1984).

Las casas sufrieron mucho. Dos buques y un bergantín se hundieron en La Unión, El Salvador. En el mar se vivió una situación dramática, dos canoas se dañaron (Montessus de Ballore, 1888; Milne, 1912; Heck, 1947; Larde, 1960; Iida et al., 1967: Sutch, 1981; Soloviev\&Go, 1984).

1859-1209 El mar estuvo muy agitado en puerto Acajutla (El Salvador). Se produjo una gran ola y un ruido terrible. El mar se retiró lejos de la costa, el nivel del agua en los embarcaderos y ríos descendió notablemente. Cavernas y grutas se derrumbaron. El rompeolas y la aduana fueron inundados. Los peces quedaron sobre la playa y las terrazas (Perry, 1862, Montessus de Ballore, 1888, Milne, 1912, Heck, 1947, Montandon, 1962; Iida et al., 1967; Soloviec \& Go, 1984).

Una onda sísmica (pudo haber sido un temblor o un tsunami) destruyó iglesias, edificios estatales y casas de habitación en Panamá (Milne, 1912; Sieberg, 1932; Iida et al., 1967; Sotoviev \& Go, 1984).

Se formaron 3 promontorios de arena de unos $2 \mathrm{~km}$ de largo en Ocos, Guatemala. Las ondas visibles en el suelo tenían 25-30 metros de largo y 25-30 cm de alto (Sapper, 1902, 1905; Montessus de Ballore, 1906; Anderson, 1908; Sieberg, 1932; lida et al-, 1967; Soloviev \& Go, 1984).

La costa de Ahuachapán en El Salvador, desde Garita Palmera hasta Barra de la Paz y más allá, fue inundada. Se escuchó un fuerte estruendo como disparos de cañón. El agua del mar se retiró y el fondo del mar quedó expuesto por una considerable distancia. Una gran ola surgió del mar y alcanzó la costa matando a 100 personas en Barra de Santiago y 85 más en Barra de la Paz. Casas y árboles fueron arrastrados hacia el mar. Se observaron tres grandes olas (Sapper, 1902, 1905; List,1903; Montessus de Ballore, 1906,: Anderson, 1908; Sieberg, 1932: Iida et al., 1967; Soloviev \& Go, 1984; Ambraseys \& Adams, 1996).

Olas continuas en el lago San Cristóbal. La costa en la región de Ocos subsidió 1 m (Rockstroch, 1902; Sapper, 1902, 1905; Montessus de Ballore, 1906; Anderson, 1908; Sieberg, 1932; Iida et al., 1967; Soloviev \& Go, 1984).

El 21 de enero el barco "Ciudad de Panamá" encontró árboles y cuerpos de animales flotando sobre las aguas del océano. Esto pudo haber sido el resultado de un temblor que ocurrió el día anterior (González, 1910; Soloviev \& Go, 1984; Ambraseys \& Adams, 1996). ola, casas arrasadas y destruidas. El tsunami fue observado a lo largo de toda la costa pacífica de América Central, así como en México y California. Una playa de $2 \mathrm{~km}$ de largo se secó en Bahía Potrero, el agua se abalanzó tierra adentro lanzando los botes hacia arriba (Sieberg, 1932; Shepard et al., 1950; lida et al., 1967; Soloviev \& Go, 1984). 


\begin{tabular}{lll}
\hline$\#$ & Fecha & Daños, efectos, comentarios \\
\hline 14 & 1906-... & $\begin{array}{l}\text { Grandes olas invadieron la playa Los Negritos. Un tsunami fue observado en esta playa (Larde, 1960; } \\
\text { Soloviev \&Go, 1984). }\end{array}$
\end{tabular}

Extraño comportamiento del mar, semejante a agua hirviendo. Repentina elevación del nivel del mar. Los ríos aumentaron su caudal inundando áreas en tierra. La villa Pedasi desapareció (MacDonaId \& Jhonston, 1913; Feldman, 1984; Viquez \& Camacho, 1993; Ambraseys \& Adams, 1996).

$1915-0907$

1916-0131

$1916-0525$

1919-0629

1919-1212

1920-1206

1926-1105

1934-0718

1941-1205

1941-1206

$1950-1005$

1950-1023

1951-0803

1952-0513

$1956-1024$
Tan solo se reporta un disturbio en el mar ( Soloviev \& Go, 1984).

Cambio del nivel del mar en la zona del Canal de Panamá (Kirkpatrick, 1920; Soloviev \& Go, 1984).

Tanto el temblor como el tsunami no son confiables (Hatori, 1995).

Areas inundadas en golfo de Fonseca y Corinto (Molina, 1997, Fernández et al., 2000).

Areas inundadas en Ostial, Nicaragua (Molina, 1997, Fernández et al., 2000).

Tsunami y/o reporte macrosísmico (Molina, 1997, Fernández et al., 2000).

El temblor fue reportado como destructivo en Nicaragua. Una sacudida fue sentida en dos barcos que se encontraban en alta mar (Leeds, 1974; Ambraseys \& Adams, 1996).

Una ola inundó la costa oeste del golfo de Chiriquí. Las casas fueron arrasadas en puerto Armuelles. El tsunami causó menor daño a lo largo de la costa oeste del golfo. Una fuerte ola fue sentida en varios barcos. Dos grandes boyas ancladas fueron desplazadas 300 metros desde su sitio original. La calle principal de la villa indígena de puerto Armuelles se inundó varias veces y las casas fueron completamente anegadas (Soloviev \& Go, 1984; Camacho, 1991; Ambraseys \& Adams, 1996).

La sacudida fue sentida a bordo de un buque. La oscilación del nivel del mar fue $23 \mathrm{~cm}$ según un mareógrafo de Puntarenas, Costa Rica. Se observaron fluctuaciones del nivel del mar en Dominical (Soloviev \& Go, 1984; Ambraseys \& Adams, 1996).

Oscilaciones del nivel del mar registradas en Puntarenas (Soloviev \& Go, 1984).

Pequeñas oscilaciones del nivel del mar registradas en San Juan del Sur, Nicaragua y La Unión, El Salvador. El mareógrafo de Puerto Armuelles fue destruido y en aquellos de Puntarenas (Costa Rica), La Unión (El Salvador), Hilo (Hawaii) y San Juan del Sur (Nicaragua) registraron una ola de $10 \mathrm{~cm}$ de alto (Murphy \& Ulrich, 1952; Iida et al., 1967; Cruz \& Wyss, 1983; Ambraseys \& Adams, 1996).

Una ola de aproximadamente $30 \mathrm{~cm}$ de alto fue registrada en San José, Guatemala. En La Unión y en Hilo la altura de la ola fue $10 \mathrm{~cm}$ (Murphy \& Ulrich, 1952; lida et al., 1967; Ambraseys \& Adams, 1996).

Fuertes temblores abrieron los lados del volcán Cosigüina y toneladas de agua bajaron por los flancos inundando el puerto de Potosí en la costa Pacífica de Nicaragua. Aparentemente, la corriente de lodo llegó al mar y provocó el tsunami (Leeds. 1974).

Una ola de 10 cm fue registrada en Puntarenas, Costa Rica (Iida et al., 1967; Soloviev \& Go, 1984).

La información sobre el temblor es confiable no así la del tsunami (Rothe, 1957; Hansen, 1972: Leeds, 1974; Ambraseys \& Adams, 1996). 
continuación...

\begin{tabular}{|c|c|c|}
\hline \# & Fecha & Daños, efectos, comentarios \\
\hline 31 & $1957-0310$ & $\begin{array}{l}\text { Una ola de varios metros de alto dañó parte del Puerto de Acajutia en El Salvador y causó perdida de } \\
\text { vidas. El temblor que provocó esta ola se originó en las Islas Aleutianas (Alvarez, 1979). }\end{array}$ \\
\hline 32 & $1960-0522$ & Tsunami originado en Chile, afectó toda la cuenca del Pacífico (Okal, 1994) \\
\hline 33 & $1962-0312$ & $\begin{array}{l}\text { Un pequeño tsunami fue registrado en San Cristóbal, Islas Galápagos. En Puerto Armuelles la ola } \\
\text { alcanzó } 30 \mathrm{~cm} \text { (Lander \& Cloud, 1964; lida et al, 1967; Soloviev \& Go, 1984; Víquez \& Toral, 1987). }\end{array}$ \\
\hline 34 & $1968-0925$ & $\begin{array}{l}\text { El camino a lo largo de la costa pacífica de Guatemala fue anegado por un tsunami cuya máxima ola } \\
\text { alcanzó } 1,9 \text { metros de alto (BSSA, 1969). }\end{array}$ \\
\hline 35 & $1976-0711$ & $\begin{array}{l}\text { Daño moderado en la provincia de Darién, Panamá, especialmente en las villas costeras de Jaque y } \\
\text { La Palma. En Jaque murió gente (Grases, 1990). }\end{array}$ \\
\hline 36 & $1990-0325$ & $\begin{array}{l}\text { Oscilaciones del nivel del mar (Gutiérrez \& Soley, 1991; Barquero \& Rojas, 1994, Ambraseys \& } \\
\text { Adams, 1996). }\end{array}$ \\
\hline 37 & $1992-0902$ & $\begin{array}{l}\text { El mayor tsunami de todos los conocidos en América Central. La mayor ola alcanzó 9,5 metros de } \\
\text { alto. El agua penetró } 1 \mathrm{~km} \text { tierra adentro en Masachapa. La extensión horizontal de la inundación fue } \\
\text { del orden de varios cientos de metros. Murieron } 170 \text { personas a causa de este tsunami, todos } \\
\text { nicaragüenses, pues fue la costa más afectada. En Costa Rica la máxima ola llegó a 2-4 metros y pro- } \\
\text { dujo daños a botes y pequeños puertos (Baptista et al., 1993; Ide et al. 1993; Imamura et al., 1993; } \\
\text { Fernández, et al., 1993; Satake, 1994; Camacho, 1994; Ambraseys \& Adams, 1996). }\end{array}$ \\
\hline
\end{tabular}

1976, causó muertes aunque no se sabe cuantas. Los tsunamis más trágicos en la costa pacífica son los de Guatemala-El Salvador de 1902, que mató 185 personas y el de Nicaragua de 1992 que se llevó la vida de 170 personas.

El temblor que ocasionó el tsunami de Nicaragua de 1992, es un caso especial de temblor denominado temblor tsunami (Kanamori, 1972). Son los llamados temblores lentos, cuya energía toma gran tiempo para acumularse (Okal, 1994) y cuando ocurre el temblor, este puede ser débilmente sentido por la población. Por esta razón y porque hay una gran desproporción entre el tamaño del temblor y el del tsunami, estos eventos son engañosos. De hecho así pasó en Nicaragua; el temblor no fue excesivamente grande y fue sentido muy débilmente por la población, por lo que no hubo la más mínima sospecha de la ocurrencia de un tsunami y la gente permaneció confiada. Este tipo de temblores es un serio problema a la hora de emitir una alerta contra tsunamis y constituyen un desafío para todos los sistemas de alerta en el mundo, ya que el potencial tsunamigénico no puede inferirse de la magnitud del temblor.

\section{ESTIMACIÓN EMPÍRICA DE LA AMENAZA}

Ambas costas han sido golpeadas por los tsunamis y en ambas costas ha habido reportes de extensiva destrucción y pérdida de vidas. Sin embargo, la costa pacífica tiene mayor amenaza que la caribe. Esto es sustentado no solo por el número de tsunamis sino por la distribución de ellos y la de los temblores que los originaron. Hay 37 temblores tsunamigénicos bien distribuidos a lo largo de esta costa (zona tsunamigénica del Pacífico), en contraste con 12 de la costa caribe que, además, se concentran cerca del golfo de Honduras (zona tsunamigénica golfo de Honduras) y de la frontera entre Panamá y Costa Rica (zona tsunamigénica Costa Rica Panamá Caribe). De esto se desprende que la amenaza más crítica está relacionada con temblores de subducción de la región centroamericana. 
En la costa caribe hay 44 centros de población expuestos a los tsunamis, 16 en Panamá, 4 en Costa Rica, 7 en Nicaragua, 9 en Honduras, 1 en Guatemala y 6 en Belice. Los puertos más importantes de esta costa son: Colón, Limón, Bluefields, puerto Cabezas, puerto Lempira, Trujillo, La Ceiba, puerto Cortés y Belice. Como se muestra en la figura 3 , los tsunamis de esta costa están concentrados en dos distintas áreas: (i) Cerca de las costas de Costa Rica y Panamá y (ii) En el

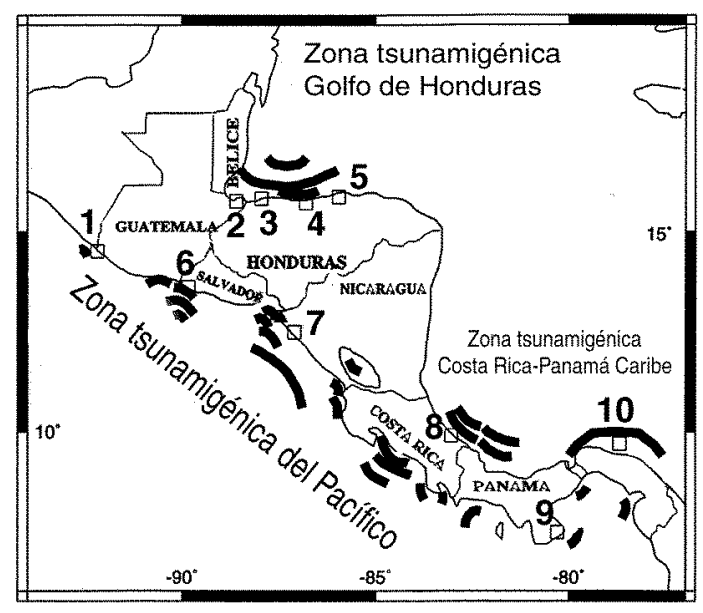

Fig. 3: Area de daño de los tsunamis (líneas gruesas). Se indican las tres zonas tsunamigénicas de la región. 1. Ocos, 2. Puerto Barrios, 3. Puerto Cortés, 4. La Ceiba, 5. Trujillo, 6. Acajutla, 7. Corinto, 8. Limón, 9. Pedasi, 10. Islas San Blas.

área del golfo de Honduras. Los temblores que generan tsunamis en estas áreas vienen del sistema de fallas Polochic-Motagua y del Cinturón Deformado de Panamá, respectivamente. Debido a que la costa caribe de Nicaragua está lejos de tales rasgos tectónicos, es menos probable que ella sea golpeada por tsunamis. Las localidades más seriamente dañadas por tsunamis en esta costa son: Omoa, Cortés, Trujillo (todas de Honduras) y las islas San Blas de Panamá. Tanto el golfo de Honduras como las costas de Panamá y Costa Rica han sido afectadas por al menos tres tsunamis. Uno de estos tuvo una ola de $5 \mathrm{~m}$ de alto y otros dos contaron con olas que llegaron a $\operatorname{los} 3 \mathrm{~m}$. Ellos devastaron villas y de acuerdo a los reportes existentes, mataron a 65 personas. Considerando 10 tsunamis de los dos últimos si- glos, la recurrencia de los temblores tsunamigénicos del Caribe es 20 años.

En la costa pacífica hay 60 centros de población expuestos a los efectos de los tsunamis. 14 en Panamá, 19 en Costa Rica, 9 en Nicaragua, 3 en Honduras, 6 en Salvador y 8 en Guatemala. De estas localidades 11 son puertos importantes, a saber: Balboa, Puerto Armuelles, Golfito, Quepos, Caldera, Puntarenas, Corinto, La Libertad, Acajutia, San José y Champerico. Las localidades más afectadas por tsunamis son: golfo Dulce, destruida por el tsunami de 1854, Acajutla que fue inundada durante los tsunamis de 1859 y 1957, Pedasi la cual desapareció después del tsunami de 1913 y el Tránsito, completamente destruida por el tsunami de Nicaragua de 1992.

En la figura 3 se aprecia que todos los países de América Central han experimentado el efecto de un tsunami. La mayoría de los pueblos costeros han sido golpeados por tsunamis locales y algunos de ellos por más de tres. El segmento costero que va desde Nicaragua hasta Guatemala parece ser la zona tsunamigénica más peligrosa no sólo por el número de tsunamis, sino por la destrucción que ellos han causado allí. En Costa Rica y en Panamá los tsunamis son escasos, pero a pesar de ello, un tsunami de Costa Rica y otro de Panamá destruyeron pequeñas villas lo cual sugiere que ellos no deben ser subestimados. La frecuencia promedio de los tsunamis de esta costa es 3,7 años.

Respecto a la magnitud de los temblores es importante anotar que aún temblores con magnitud 6 han generado tsunamis en ambas costas de América Central. Un temblor de magnitud 6 disparó el tsunami de 1913 que destruyó Pedasi y otro generó una ola de más de un metro en la costa caribe. Sin embargo, los más grandes y más destructivos tsunamis estuvieron asociados con sismos de magnitud mayor o igual a 7. Temblores regionales de magnitudes superior a 8 produjeron tsunamis que alcanzaron las costas de América Central.

El tsunami de Nicaragua de 1992 es el más grande de América Central. De este evento se aprendieron dos importantes lecciones: (i) Un temblor no muy grande puede generar un gran tsunami y (ii) la falta de grandes tsunamis históricos 
no necesariamente significa que ellos no ocurrirán alguna vez en el futuro. Considerando lo último, es posible que un gran tsunami pueda ocurrir en áreas donde no ha habido tsunamis previamente. Esta posibilidad representa una amenaza potencial, especialmente si se toma en cuenta la expansión de la actividad turística a lo largo de las costas centroamericanas.

\section{CONCLUSIONES}

De acuerdo con esta investigación, han habido 49 tsunamis en América Central desde 1539 hasta el presente, 12 son en la costa caribe y 37 en la pacífica; 34 están bien documentados. Estos han destruido localidades y matado al menos 455 personas. A lo largo de ambas costas ha habido varios tsunamis con olas de amplitud superior a $3 \mathrm{~m}$ y dos con amplitud mayor a $5 \mathrm{~m}$. La mayor de todas estas olas corresponde al tsunami de Nicaragua que alcanzó 9,5 m.

En la costa caribe los tsunamis se concentran en el golfo de Honduras y frente a las costas de Panamá y Costa Rica. Ellos están relacionados con la actividad sísmica en el sistema de fallas PolochicMotagua y en el Cinturón Deformado de Panamá. En el Pacífico se distribuyen a lo largo de toda la costa desde Guatemala hasta Panamá y están asociados con temblores de subducción. El segmento costero que se extiende desde Nicaragua hasta Guatemala es la sección con más alta probabilidad de ser golpeada por los tsunamis más grandes.

Los temblores con mayor probabilidad de generar grandes tsunamis son aquellos de magnitud mayor o igual a 7,0, localizados en el piso oceánico. En el Pacífico, $43 \%$ de estos eventos generan tsunamis mientras que en el Caribe el $100 \%$ de ellos generaron pequeños tsunamis el pasado siglo. Otros temblores de otras áreas del Cinturón de Fuego del Pacífico requieren magnitudes mayores a 8 para generar tsunamis que afecten las costas de América Central.

Considerando los históricos y recientes tsunamis de América Central y tomando en cuenta el crecimiento de las localidades costeras debida a la actividad turística, un efectivo sistema de alerta contra tsunamis es necesario en América Central.

\section{REFERENCIAS}

ALVAREZ, S., 1979: Apuntes de sismología general y normas de prevención de terremotos. - 46 págs. Centro Invest. Geotéc. El Salvador [Inf. interno].

AMBRASEYS, N., 1995: Magnitudes of Central America earthquakes, 1898-1930. - Geophys. J. Int., 121: 545546.

AMBRASEYS, N. \& ADAMS, R., 1996: Large magnitude Central America earthquakes, 1898-1994. Geophys. J. Int. 127: 665-692.

ANDERSON, T., 1908: The volcanoes of Guatemala. Geogr. J. 31: 473-489.

BAPTISTA, A., PRIEST, G. \& MURTY, T., 1993: Field Survey of the 1992 Nicaragua Tsunami. - Marine Geodessy, 16: 169-203.

BARQUERO, R. \& ROJAS,W.,1994: Sismicidad inducida por el terremoto de Limón. - Rev. Geol. América Central, vol. esp. Terremoto de Limón: 111-120.

BOSCHINI, I. \& MONTERO, W.,1994: Sismicidad histórica e instrumental del Caribe de Costa Rica. - Rev. Geol. América Central, vol. esp. Terremoto de Limón: 65-72.

BOWIN, C., 1976: The Caribbean gravity field and plate tectonics. - Geol. Soc. Amer. Spec. Paper, 169: 1-79.

BSSA, 1969: Seismological notes Mexico-Guatemala border, April 9-10, 1968.- Bull. Seis. Soc. Amer. 59(2): 1028.

BUCKMAN, R., PLAFKER, G. \& SHARP, R., 1978: Fault movement following the Guatemala earthquake of February 4, 1976. - Geology, 6: 170-173.

CALAIS, E., BETHOUX, N. \& MERCIER DE LEPINAY, B., 1992: From transcurrent faulting to frontal subduction: A seismotectonic study of the northern Caribbean plate boundary from Cuba to Puerto Rico. Tectonics, 11(1): 114-123.

CAMACHO, E., 1991: The Puerto Armuelles earthquake (Southwestern Panama) of July 18, 1934. - Rev. Geol. América Central, 13: 1-14.

CAMACHO, E., 1994: El tsunami del 22 de abril de 1991 en Bocas del Toro, Panamá. - Rev. Geol. América Central, vol. esp. Terremoto de Limón: 61-64.

CAMACHO, E. \& VÍQUEZ, V., 1992: Historical seismicity of the North Panama Deformed Belt. - 50 págs Inst. Geoci., Univ. de Panamá [Inf. interno]. 
CAMACHO, E. \& VÍQUEZ, V., 1993: Historical seismicity of the North Panama Deformed Belt. Rev. Geol. América Central, 15: 49-64.

CARR, M. \& STOIBER, R., 1977: Geological setting of some destructive earthquakes in Central America, Appendix I: Destructive earthquakes in Central America. - Bull. Geol. Soc. Am. 88: 151-156.

CRAWFORD, J., 1902: Earthquakes in Nicaragua. - Amer. Geologist, 29, 323.

CRUZ, G. \& WYSS, M., 1983: Large earthquakes, mean sea level, and tsunami along the Pacific Coast of Mexico and Central America. - Bull. Soc. Seism. Am. 73: 553-570.

DENYER, P., ARIAS, O. \& PERSONIUS, S., 1992: Generalidades sobre los efectos geológicos del terremoto de Limón. - Rev. Geol. América Central, vol. esp. Terremoto de Limón: 29-38.

FELDMAN, L., 1984: A Catalogue of historical documents pertaining to the earthquake damage in Panama and Costa Rica. [Unpubl. report].

FERNÁNDEZ, M., HAVSKOV, J. \& ATAKAN, K., 1999: Destructive tsunamis and tsunami warning in Central America. - Science Tsunami Hazards, 17(3): 173-185.

FERNÁNDEZ, M., MOLINA, E., HAVSKOV, J. \& ATAKAN, K., 2000: Tsunamis and tsunami hazards in Central America. - Natural Hazards, 22: 91-116.

FERNÁNDEZ, M., PERALDO, G., ROJAS, W. \& FLORES, R., 1993: Tsunamis en Centroamérica. - Tecnol. en Marcha, 12(2):10-17.

GONZÁLEZ, C., 1910: Temblores, terremotos, inundaciones y erupciones volcánicas en Costa Rica, 1606-1910. 200 págs. Tipografía de Avelino Alsina, San José.

GRASES, J., 1990: Terremotos destructores del Caribe 15021990. - UNESCO-RELACIS, Caracas, Agosto de 1990.

GÜENDEL, F., 1986: Seismotectonic of Costa Rica: An analytical view of the southern terminus of the Middle America Trench. - 157 págs. Univ. de California, Santa Cruz [Ph.D. Tesis].

GUTIÉRREZ, A. \& SOLEY, J., 1991: Análisis de los registros de nivel del mar correspondientes al terremoto de Cóbano de 23-3-90. - Geofísica, 35: 182-196.

HANSEN, F., 1972: Origen de los sismos - Historia sísmica de Nicaragua. - 27 págs. 8 láminas, Managua [Inf. inédito].
HATORI, T., 1995: Magnitud Scale for the Central America Tsunamis. - PAGEOPH, 144(3/4): 471-479.

HECK, N., 1947: List of seismic waves. - Bull. Seism. Soc. Am. 37: 269-285.

IDE, S., IMAMURA, F., YOSHIDA, Y. \& ABE, K., 1993 Source characteristics of the Nicaragua tsunami earthquake of September 2, 1992. - Geophys. Res. Lett., 20: 863-866.

IIDA, D., COX, C. \& PARARAS-CARAYANNIS, G., 1967: Preliminary catologue of tsunamis occurring in the Pacific Ocean. - [sin págs]. Hawaii Inst. Geophys. Univ. Hawaii, Data Rep. N 5.

IMAMURA, F., SHUTO, N., IDE, S., YOSHIDA, Y. \& ABE, K., 1993: Estímate of the tsunami source of the 1992 Nicaragua earthquake from tsunami data. - Geophys. Res. Lett. 20: 1515-1518.

JORGENSEN, M., 1966: Seismological history of the Canal Zone and Panama, Panama Canal Company, Canal Zone. - 12 págs. IOCS Memorandun, PCC-3.

KANAMORI, H., 1972: Mechanism of tsunami earthquakes. - Phys. Earth Planet. Inter. 6: 346-359.

KIRKPATRICK, Z., 1920: Earthquakes in Panama up to January 1, 1920. - Bull. Soc. Seis. Am. 10(3): 120-128.

LANDER, J., \& CLOUD, W., 1964: U.S Earthquakes 1962. - U.S Dep. Commerce, Coast and Geodetic Surv. Washington D.C., 1-114.

LARDE, J., 1960: Historia sísmica y erupciones volcánicas de El Salvador. - En: Min. Cultura, Depto. Ed, Documentación histórica, crítica y conclusiones, Obras completas Tomo I: 441-576.

LEEDS, J., 1974: Catalog of Nicaragua earthquakes. - Bull. Soc. Seis. Am. 64: 1135-1158.

MacDONALD, F. \& JHONSTON. C., 1913: Isthmian earthquakes. - Canal Record, 7: 144-149.

MENDOZA, C., \& NISHENKO, S. 1989: The North Panama earthquake of 7 september 1882: evidence for active underthrusting. - Bull. Seismol. Soc. Am.79: 1264-1269.

LIST, K., 1903: Erdbeben an der Küste Guatemalas im Jahre 1902 und deren Folgeerscheinungen. -Ann. Hydrograph. Marit. Meteorolog. Beilag, ii-iv, Hamburg: 52-54.

MILNE: 1912, Catalogue of destructive earthquakes A.D. $7-$ 1899. - Report 81st Meeting, Br. Assoc. Adv. Sci. Portsmouth, 191: 649-670. 
MOLINA, E., 1997: Tsunami catalogue for Central América 1539-1996. - 87 págs. Univ. Bergen, Noruega [Reporte técnico II 1-04].

MONTANDON, F., 1962: Les megaseismes en Amerique: Revue pour L'Etude des Calamites. - Bull. 1'Union Internat. de Secours, 38: 57-97.

MONTERO, W., 1986: Períodos de recurrencia y tipos de secuencia sísmica de los temblores intraplaca en la región de Costa Rica. - Rev. Geol. América Central, 5: 35-72.

MONTESSUS DE BALLORE, F., 1888: Tremblements de terre et eruptions volcaniques on Centre Amerique depuis la coquete espagnole fusqu'a nos jours.- 293 págs. Soc. Sci. Naturelles de Saore-etLoire. Dijon.

MONTESSUS DE BALLORE, F., 1906: Les tremblements de Terre: Geographie Seismologique.- Librairie Armand Colín, Paris: 384-392.

MURPHY, L. \& ULRICH, F., 1952: U.S. Earthquakes, 1950, U. S. Dep. Commerce, Coast and Geodetic Surv. Washington D.C. 755: 1-47.

NELSON, W., 1889: Five years at Panama, the trans-isthmian Canal.- 287 págs. Belford, New York.

OKAL, E., 1994: Tsunami warning: beating the waves to death and destruction. - Endeavour, New Series. V, 18(1): 38-43.

PACHECO, J. \& SYKES, L., 1992: Seismic moment catalog of large, shallow earthquakes, 1900-1989. - Bull. Seism. Soc. Am. 82: 1306-1349.

PERALDO, G. \& MONTERO, W., 1994: Los temblores del periodo colonial de Costa Rica, 1538-1821. - 162 págs. Ed. Tecnol. de Costa Rica, Cartago.

PERRY, M., 1855-1856, 1861-1862): Bibliographie Seismique.- Academie de Dijon, iv-v (1855-56) and ix-x (1861-62). Dijon.

PLAFKER, G. \& WARD. S., 1992: Backarc thrust faulting and tectonic uplift along the Caribbean sea coast during April 22, 1991 Costa Rica Earthquake. - Tectonics, 11: 709-718.

ROCKSTROCH, E., 1902: Earthquake in Guatemala. - Nature, 66: 150.
ROJAS, W., BUNGUN, H. \& LINDHOLM, C., 1993: A catalog of historical and recent earthquakes in Central America. - 78 págs. NORSAR, Oslo, Noruega, [Technical report].

ROTHE, J., 1957: Chronique seismologique. - Revue por l'Etude des Calamites, Geneva, 36: 2-27.

SATAKE, K., 1994: Mechanism of the 1992 Nicaragua tsunami earthquake. - Geophys. Res. Lett., 21(23): 2519-2552.

SAPPER, K., 1902: Das Erdbeben in Guatemala vom 18 April 1898 und die Maribios-Vulkane.- Globus, 75: 201-208.

SAPPER, K., 1095: In den Vulkangebieten Mittelamerikas und Westindiens. Reiseschilderungen und Studien über die Vulkanausbrüche der Jahre 1902 bis 1903, ihre geologischen, wirtschaftlichen und sozialen Folgen. - 334 págs. E. Schweizerbart, Stuttgart.

SHEPARD, F., McDONALD, G. \& COX, L. 1950: The tsunami of April, 1946.- Bull. Scripps Inst. Oceanog. 5:391-528.

SIEBERG, A., 1932: Erdbebengeographie: Mittelamerika samt Westindien.- Handbuch der Geophysik. IV(B): 948-960.

SOLOVIEV, L. \& GO, N., 1984: A catalogue of tsunamis on the eastern shore of the Pacific Ocean (1513-1968).204 págs. Nauka Publishing House, Moscow.

SUTCH, L., 1981: Estimated intensities and probable tectonic sources for the historic (pre-1898) earthquakes.Bull. Soc. Seism. Am. 71: 865-881.

TABOR, L., 1967: Compilation on seismic data: Panama and adjoining and sea area, NVO-99-22. - 40 págs. Blume, San Francisco.

VÍQUEZ, V. \& TORAL, J., 1987: Sismicidad histórica sentida en el istmo de Panamá. - Geofísica, 27: 135-165.

VÍQUEZ, V. \& CAMACHO, E., 1994: El terremoto de Panamá La Vieja del 2 de mayo de 1621: un sismo intraplaca. - Bol. Vulcanol. 23: 13-20.

YOUNG, C., LAY, T. \& LYNNES, C., 1989: Rupture of the 4 February 1976 Guatemalan earthquake. - Bull. Seis. Soc. Am. 79: 670-689. 